\title{
Self-organized criticality in stick-slip models with periodic boundaries
}

\author{
Kwan-tai Leung ${ }^{1}$, Jørgen Vitting Andersen ${ }^{2}$ and Didier Sornette ${ }^{3,4}$ \\ ${ }^{1}$ Institute of Physics, Academia Sinica, Taipei, Taiwan 11529, R.O.C. \\ ${ }^{2}$ Department of Mathematics, Imperial College, Huxley Building, 180 Queen's Gate, London SW7 2BZ, U.K. \\ ${ }^{3}$ Laboratoire de Physique de la Matière Condensée, CNRS, Université de Nice-Sophia Antipolis, \\ Parc Valrose, 06108 Nice Cedex 2, France \\ ${ }^{4}$ Department of Earth and Space Sciences and Institute of Geophysics and Planetary Physics \\ University of California, Los Angeles, California 90095-1567, U.S.A.
}

(Last revised December 5, 2017)

\begin{abstract}
A spring-block model governed by threshold dynamics and driven by temporally increasing spring constants is investigated. Due to its novel multiplicative driving, criticality occurs even with periodic boundary conditions via a mechanism distinct from that of previous models. This mechanism is dictated by a coarsening process. The results show a high degree of universality. The observed behavior should be relevant to a class of systems approaching equilibrium via a punctuated threshold dynamics.
\end{abstract}

PACS numbers: 64.60.Lx, 64.60.-i, 05.70.Jk

Out-of-equilibrium driven systems with threshold dynamics exhibit a rich phenomenology, from synchronized behavior [1,2] to self-organized criticality (SOC) [3,5]. SOC refers to the spontaneous organization towards a kind of dynamical critical steady state. Threshold out-ofequilibrium dynamics encompasses many systems, such as neural networks, solid friction, rupture with healing, earthquakes and avalanches. It is now understood qualitatively that there is a class of models exhibiting SOC as a result of their tendency to synchronize [6]. This tendency is however frustrated by constraints such as open boundary conditions [6] and quenched disorder [7] which leads to a dynamical regime at the edge of synchronization, the SOC state. Another class, so-called extremal models, are understood to exhibit SOC due to the competition between local strengthening and weakening due to interactions [8]. In a third class of models, SOC results from the tuning of the order parameter of a system exhibiting a genuine critical point to a vanishingly small, but positive value, thus ensuring that the corresponding control parameter lies exactly at its critical value for the underlying depinning transition [9]. The issue is furthermore complicated by the fact that a notable fraction of numerical and experimental works 10 12 claiming the observation of SOC from the measurements of powerlaw distributions rely on the slow sweeping of a control parameter towards a critical point [13.14].

The purpose of this letter is to present a variation of spring-block models using a novel form of driving by multiplicative loading. The surprising result is that, when the dynamics is conservative and for periodic boundary conditions ( $\mathrm{PBC})$, the system self-organizes into a critical state with long-range order and power-law distributions. This is in contrast to all previous stick-slip models [4, 6, 15 that need either open boundary conditions and/or dissipation to exhibit SOC (in order for synchronization not to occur). None of the four above mechanisms seem at work here. We conjecture that this new class appears as a result of the multiplicative nature of the driving, known in simpler models to lead to stationary dynamical states with power law distributions [16]. Our model exhibits the dual property of approaching (without ever reaching) an equilibrium state in terms of block positions while at the same time being characterized by a steady state in the force variables. To our knowledge our model is the first of its kind to show SOC in approach to an equilibrium state, and as such suggests a new class of experimental systems which could exhibit SOC states.

Model: We consider a two-dimensional spring-block model consisting of an array of blocks interconnected among nearest neighbors by coil springs. The springs have the same spring constant $K$ and relaxed spring length $l$. Initially, the array is stretched to a lattice spacing $a>l$ and placed on a frictional substrate which is characterized by a static threshold $F_{s}$ for slipping. Disorder is introduced in the form of random displacements $(x, y)$ of the blocks about the coordinates $(i a, j a)$ on a square lattice, where $-A \leq x, y \leq A$ and $i, j=1, \ldots, L$. The force between two neighboring blocks at $\vec{r}$ and $\overrightarrow{r^{\prime}}$ is given by the Hooke's law $\left(\left|\vec{r}-\overrightarrow{r^{\prime}}\right|-l\right) K$. Since we are interested in dynamics primarily governed by tensile stresses, this nonlinear dependence on the coordinates leads to unnecessary complications in the algorithm. To simplify and compare with similar models, we expand the expression to first order in $(x, y)$ to obtain the force components on a block in the bulk at $(i, j)$ 17.

$$
\begin{gathered}
F_{i, j}^{x}=\left(x_{i+1, j}+x_{i-1, j}-2 x_{i, j}\right) K \\
\quad+\left(x_{i, j+1}+x_{i, j-1}-2 x_{i, j}\right) s K \\
F_{i, j}^{y}=\left(y_{i+1, j}+y_{i-1, j}-2 y_{i, j}\right) s K \\
\quad+\left(y_{i, j+1}+y_{i, j-1}-2 y_{i, j}\right) K,
\end{gathered}
$$


where $s=1-l / a>0$ is the initial strain. It is important to stress that the terms proportional to $s$ leads to anisotropic couplings to nearest neighbors in the transverse direction. The coupling and the SOC state disappears for $s=0$ or in 1D-chains.

Since the forces are linear in displacements, it is possible to invert (11) and formulate the model solely in terms of force variables, as in [4]. Starting with a stable configuration with net force $F \equiv \sqrt{F^{x^{2}}+F^{y^{2}}}<F_{s}$ for all the blocks, we drive the system by gradually increasing $K$ 18 until one of the blocks becomes unstable, i.e., $K$ is increased to $K F_{s} / F_{\max }$ during loading, where $F_{\max }$ denotes the spatial maximum of $F$ in the stable configuration. As in [4], the block is assumed to slip to its equilibrium position defined by $F=0$, ignoring overshot:

$F_{i, j}^{x} \rightarrow 0, \quad F_{i, j}^{y} \rightarrow 0$

$F_{i \pm 1, j}^{x} \rightarrow F_{i \pm 1, j}^{x}+\alpha F_{i, j}^{x}, \quad F_{i \pm 1, j}^{y} \rightarrow F_{i \pm 1, j}^{y}+s \alpha F_{i, j}^{y} ;$

$F_{i, j \pm 1}^{x} \rightarrow F_{i, j \pm 1}^{x}+s \alpha F_{i, j}^{x}, \quad F_{i, j \pm 1}^{y} \rightarrow F_{i, j \pm 1}^{y}+\alpha F_{i, j}^{y}$,

where $\alpha=1 /(2+2 s)$. This locally conserves the force components, thus the conservative nature of the model. The resulting modification of the stress environment may trigger further slips in neighboring blocks, and hence an avalanche, until $F<F_{s}$ is restored for all blocks. Then $K$ is increased again and the slip process continues.

If $F_{s}$ was zero, the only stable (minimal energy) configuration would be that the blocks were exactly at the nodes of a perfect square lattice of mesh size $a$. The non-vanishing friction thus creates a large ensemble of coexisting metastable states which is responsible for the nontrivial dynamics. For $F_{s} \neq 0$ and $s \neq 0$, the toppling rules (2) do not put the blocks in their minimum energy configuration due to the couplings to their four neighbors. This ensures that a block will go on becoming unstable ad infinitum as long as $K$ is increased indefinitely.

The multiplicative loading is motivated by the stiffening of an overlayer caused by desiccation 19, originally used to study cracks [20]. It differs from the usual additive loading in sandpiles [3] 6] and stick-slip models 15.17 where physically the driving force arises from the dropping of grains onto a pile or from the steady relative motion of two frictional surfaces. Those systems are known to exhibit self-organized criticality in non-equilibrium steady states. In contrast, our system approaches an equilibrium instead of a genuine steady state.

Without loss of generality, we hereafter set $a=1=F_{s}$. Of the remaining dimensionless parameters $\{s, A, K\}, s$ determines the equilibrium length scale and the dynamics through (2), $A$ characterizes the initial disorders but is irrelevant for the equilibrium state, and $K$ defines the "time" $t \equiv K$.

Results: We have simulated the model for $0 \leq s \leq 1$, $A=0.02,0.18,0.4$, and system sizes $20 \leq L \leq 300$. We are mainly interested in the possibility of SOC with PBC as this most differentiates us from previous works. Unless stated otherwise, PBC is assumed hereafter. To investigate the effects of spatial inhomogeneities, we also use free boundary conditions (FBC) with no block beyond the edges and corners, and cylindrical boundary conditions $(\mathrm{CBC})$ with one pair of parallel edges periodic and the other pair free. All these boundary conditions respect the conservation law of the force component.

The evolution of the system is monitored by the variation of the stress field $\sigma(\vec{r}, t)$, approximated by the averaged tension of the four springs attached to a block. Using its Fourier transform $\tilde{\sigma}$, we compute the structure factor $\mathcal{S}(\vec{k}, t)=\left\langle|\tilde{\sigma}(\vec{k}, t)|^{2}\right\rangle / L^{2}-L^{2} \delta_{\vec{k}, 0}\langle\overline{\tilde{\sigma}(\vec{k}, t)}\rangle^{2}$ and the circular average $\mathcal{S}_{\text {cir }}(k, t)=\sum_{\vec{k} \in\{|\vec{k}|=k\}} \mathcal{S}(\vec{k}, t)$, where the overline and the angular brackets mean a spatial and an ensemble average, respectively. Then $R(t) \equiv\left[\sum_{k} k^{2} \mathcal{S}_{\text {cir }}(k, t) / \sum_{k} \mathcal{S}_{\text {cir }}(k, t)\right]^{-1 / 2}$ gives a simple measure of the characteristic length for the stress field.

The system evolves under a conservative dynamics from large disorders to small disorders when the blocks converge onto a perfect lattice. This is analogous to spinodal decomposition 21 and suggests a coarsening in the stress field. We indeed find a power-law growth $R(t) \sim t^{\phi}$, where $\phi=0.33 \pm 0.01$ (cf. [20]) for all $s$ and $A$, as illustrated in Fig. 1. This universal behavior and the value of $\phi$ agree with spinodal decomposition.

Now we present the evidence showing the system in the long-time limit is stationary and critical in the variables relevant to the dynamics. Fig. 1 shows the first evidence of stationarity in $R(t)$, where it reaches a plateau after transcient. While the saturation rate depends on $s$ and $A$, the value of the plateau $R_{0}$ depends only on $s$ due to $s L$ being the length the system has to contract to reach a stress-free state. But this cannot be achieved due to PBC, so that the blocks wind up in a frustrated state correlated over this distance. For fixed $L$, we verify that $R_{0}(s, L)$ increases linearly in $s$, except very close to $s=0$ or 1 . The plateau extends up to ten decades in $t$, until reaching the limit of numerical accuracy (i.e., $10^{-15}$ in $F$, using double precision). Further evidence comes from $\bar{F}(t) / F_{s}$ which measures the effective "distance" from the instability limit for the system. The stationary fluctuations of this ratio about a finite constant, as shown in Fig. 2, is an important characteristic of a dynamical steady state in SOC models [3].

Next, we show that the system is critical. Firstly, for fixed $s$, we find $R_{0}(s, L) \propto L$, implying long-range correlations in the stress field. More importantly, $R$ satisfies finite-size scaling $R(t, L)=t^{\phi} \tilde{R}\left(t / L^{1 / \phi}\right)$ (see Fig. 3), with the asymptotic behavior $\tilde{R}(x \rightarrow 0)=$ const and $\tilde{R}(x \rightarrow \infty) \propto x^{-\phi}$. This is a clear signature of criticality.

Secondly, the avalanches are characterized by powerlaw distributions, another hallmark of criticality. From (1), the force drop in one block-slip is given by $F^{\text {slip }}=$ $K u / \alpha$ [4], where $u$ denotes the slip distance. Since $F^{\text {slip }} \gtrsim F_{s}, u$ diminishes as $1 / K$ when the blocks gradually converge to a regular lattice. An avalanche consists 
of a correlated sequence of $S$ block-slips. Analogous to seismology [22], the "seismic moment" $M$ and the "radiated seismic energy" $E$ can be computed:

$$
\begin{aligned}
& M=K \sum_{l=1}^{S} u_{l}=\alpha \sum_{l=1}^{S} F_{l}^{\text {slip }} \gtrsim \alpha F_{s} S, \\
& E=\frac{1}{2} \sum_{l=1}^{S} F_{l}^{\text {slip }} u_{l}=\frac{\alpha}{2 K} \sum_{l=1}^{S}\left(F_{l}^{\text {slip }}\right)^{2} \gtrsim \frac{F_{s} M}{2 K} .
\end{aligned}
$$

These can be measured experimentally, as is precisely done for earthquakes. In our context, $E$ equals the energy dissipated by friction. We find that the distribution of $S$ approaches a power law $P(S) \sim S^{-(B+1)}$ with $B=0.15 \pm 0.05$ (see Fig. 4), when $R(t, L) \rightarrow R_{0}(L)$ for $t \gtrsim L^{1 / \phi}$. Consequently, both distributions of $M$ and the scaled energy $E K$ are stationary and follow the same power law. From these results, we conclude that our system approaches in a punctuated manner without ever reaching its final equilibrium, and is driven into a marginally stable state which is critical in physical variables.

The approach to thermodynamic equilibrium via spinodal decomposition 21 differs from our case in that its dynamics is governed by thermal fluctuations, not a threshold. On the other hand, it is well known that all previous SOC models 3 [7] constructed with threshold dynamics do not exhibit SOC with $\mathrm{PBC}$, because the periodic boundaries forbid an outflux of the conserved quantity (the total number of grains or the total force on the array) to balance its external influx. The key difference with our model is that the variables $F_{x}$ and $F_{y}$ which are redistributed conservatively can be of either sign, with the total force remaining at zero during both block slips and loading, so that $F$ which determines topplings (i.e., slips) is neither monotonic nor conserved. This separation of the conservation law from the toppling condition allows for criticality despite PBC. Another way to rationalize our results is to notice that, as mentioned above, the mechanisms at work to produce powerlaws in the presence of multiplicative noise (amplification by multiplication followed by reinjection) [16] might also be relevant here.

We also test the robustness of the critical properties against boundary effects. We find the same exponent $B$ within numerical uncertainty for $\mathrm{PBC}, \mathrm{FBC}$, and $\mathrm{CBC}$. This is remarkable in view of their different equilibrium states and paths of approach, and the usual sensitive dependence of SOC systems on boundary conditions.

Our model depends significantly on only one factor: the conservation. To show this, we introduce nonconservation by adding a term $-(x, y) \kappa K$ to $\left(F_{x}, F_{y}\right)$ in (11), which may represent harmonic couplings of each block with another surface, as in earthquake models [4]. The parameter $\kappa$ then quantifies the level of nonconservation, with $\alpha$ in (2) replaced by $1 /(2+2 s+\kappa)$ [1.17]. For not too small $\kappa(\gtrsim 0.1), \bar{F}(t) / F_{s}$ does not show any stationary regime, and $P(S)$ is exponential for all choices of sampling intervals in $t$. Consequently, the SOC state for $\kappa=0$ is lost except at most for a small interval near $\kappa=0$.

Conclusion: It has been suggested that SOC in sandpile and spring-block models arises from a de-sychronization mechanism that is initiated by inhomogeneities from a free boundary [6]. We have studied a system which shows SOC without such inhomogeneities. This reveals a different mechanism whereby correlations (or "self-organized" regions) gradually build up in the bulk via a coarsening process. The completion time $\tau \approx L^{1 / \phi}$ (cf. Fig. 3) is characterized by the coarsening exponent $\phi$, which should be compared with the invasion time in previous models. The associated power-law exponent is extremely robust (universal) with respect to the initial disorder, the initial strain, the size of the system and the type of boundary conditions. While exhibiting a well-defined steady state in the characteristic length and the average force, the model has also a transient nature when viewed in the slip distance toward the equilibrium and in the sweeping of the spring coefficient $K$. This teaches us that experimental systems that appear transiently driven might in fact be stationary in the variable relevant to the dynamics, especially when converging to a fundamental equilibrium state. Search schemes and optimization techniques using the sweeping of a control parameter such as in simulated annealing to get access to the fundamental state or to the optimal solution might exhibit this kind of phenomenon in which the relaxation is characterized by a wide distribution of jumps.

The authors wish to thank Henrik Jeldtoft Jensen for useful discussions. J.V.A. wishes to acknowledge supports from the European Union Human Capital and Mobility Program contract number ERBCHBGCT920041 under the direction of Prof. E. Aifantis. K.-t.L. is supported by the National Science Council and the NCHC of ROC. D.S. is partially supported by NSF EAR9615357.

[1] S.H. Strogatz and I. Steward, Scientific American 269, 102 (Dec, 1993).

[2] A.V.M. Herz and J.J. Hopfield, Phys. Rev. Lett. 75, 1222 (1995).

[3] P. Bak, C. Tang, K. Wiesenfeld, Phys. Rev. A 38, 364 (1988); Dhar D. et Ramaswamy R., Phys.Rev.Lett. 63, 1659 (1989); Dhar D., ibid. 64, 1613 (1990).

[4] Z. Olami, J. S. Feder, and K. Christensen, Phys. Rev. Lett. 68, 1244 (1992); K. Christensen and Z. Olami, Phys. Rev. A 46, 1829 (1992).

[5] H. Feder and J. Feder, Phys. Rev. Lett., 66, 2669 (1991); I.M. Jánosi and J. Kertész, Physica A 200, 179 (1993); J. E. S. Socolar, G. Grinstein and C. Jayaprakash, Phys. Rev. E 47, 2366 (1993); P. Grassberger, Phys. Rev. E., 49, 2436 (1994). 
[6] K. Christensen, PhD Thesis Oslo, (Nov. 1992); A. Corral, C.J. Pérez, A. Díaz-Guilera and A. Arenas, Phys.Rev.Lett. 74, 118 (1995); A.A. Middleton and C. Tang, ibid. 74, 742 (1995); S. Bottani, ibid. 74, 4189 (1995); L. Gil and D. Sornette, ibid. 76, 3991 (1996); A. Corral, C.J. Pérez, A. Díaz-Guilera, ibid. 78, 1492 (1997).

[7] D. Sornette, P. Miltenberger and C. Vanneste, Pageoph 142, 491 (1994); in "Recent Progresses in Statistical Mechanics and Quantum Field Theory", eds. P. Bouwknegt et al., (World Scientific, Singapore, 1995), p.313-332.

[8] M. Paczuski, S. Maslov and P. Bak, Phys. Rev. E 53, 414 (1996).

[9] D. Sornette and I. Dornic, Phys.Rev. E 54, 3334 (1996); D. Sornette, A. Johansen and I. Dornic, J.Phys.I France 5, 325 (1995).

[10] Cannelli G., Cantelli R. and Cordero F., Phys.Rev.Lett. 70, 3923 (1993).

[11] W. Clauss et al, Europhys. Lett. 12, 423 (1990).

[12] P.J. Cote and L.V. Meisel, Phys. Rev. Lett. 671334 (1991); J.S. Urbach, R.C. Madison and J.T. Markert, ibid. 75276 (1995).

[13] D. Sornette, Phys. Rev. Lett. 722306 (1994); J. Phys. I 4, 209 (1994).

[14] O. Perkovic, K. Dahmen and J.P. Sethna, Phys. Rev. Lett. 754528 (1995).

[15] J. Schmittbuhl, J.P. Vilotte and S. Roux, J. Geophys. Res. 101, 27741 (1996).

[16] R. Graham and A. Schenzle, Phys. Rev. A 25, 1731 (1982); A. Schenzle and H. Brand, Phys. Rev. A 20, 1628 (1979); Levy, M. and Solomon, Int. J. Mod. Phys. C 7, 65 (1996); Sornette, D. and Cont, R., J. Phys. I France 7, 431 (1997).

[17] K.-t. Leung, J. Müller, and J. V. Andersen, J Phys I France 7, 423 (1997).

[18] Although our model is driven by increasing $K$ and fixed $F_{s}$, physically only an increasing $K / F_{s}$ matters. Thus it describes the general behavior of real materials which become weaker (smaller $F_{s}$ ) as they get stiffer (larger $K$ ).

[19] A. Groisman and E. Kaplan, Europhys. Lett. 25, 415 (1994).

[20] J. V. Andersen, Y. Brechet, and H. J. Jensen, Europhys. Lett. 26, 13 (1994); J. V. Andersen, Phys. Rev. B 49, 9981 (1994); K.-t. Leung and J. V. Andersen, Europhys. Lett. (in press, 1997).

[21] See, e.g., J.D. Gunton, M. San Miguel and P.S. Sahni, Phase Transitions and Critical Phenomena, Vol. 8, ed. C. Domb and J.L. Lebowitz (Academic, NY, 1983).

[22] See, e.g., C.H. Scholz, The Mechanics of Earthquakes and Faulting (Cambridge, Cambridge, 1990).

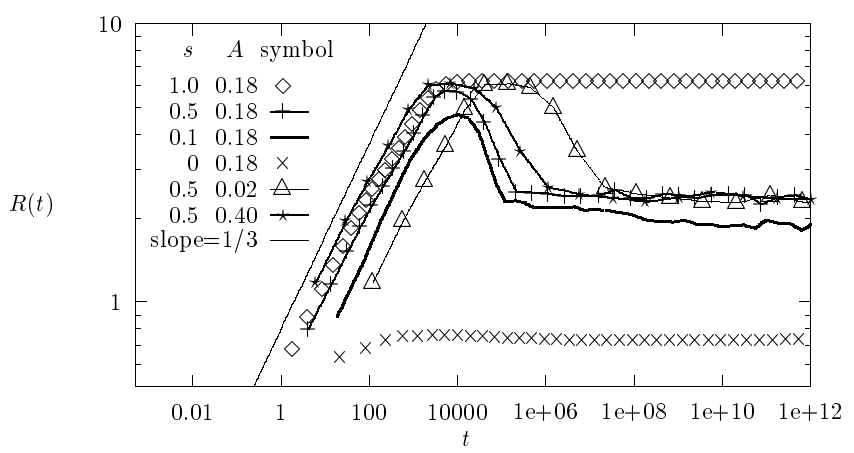

FIG. 1. Characteristic length $R(t)$ vs time $t \equiv K$ for different sets of $s$ and $A$, showing the universal $1 / 3$ power law and plateau. $L=40$ with $\mathrm{PBC}$.

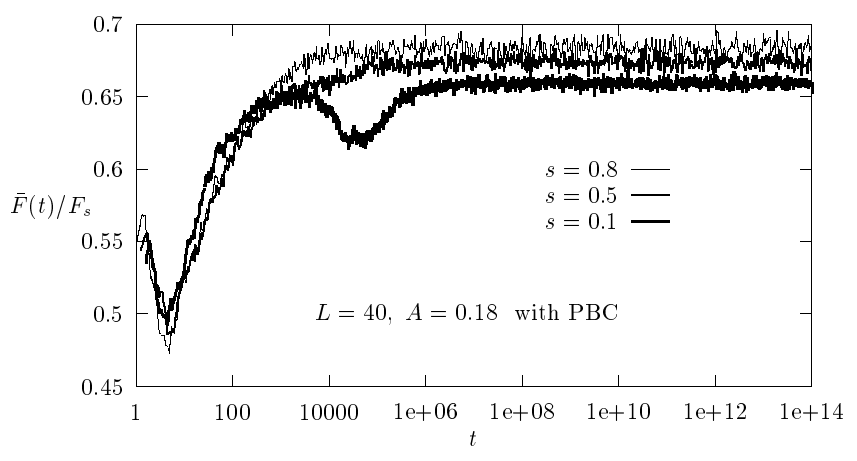

FIG. 2. Spatial averaged force on a block normalized by the threshold vs time, showing approaches to stationarity.

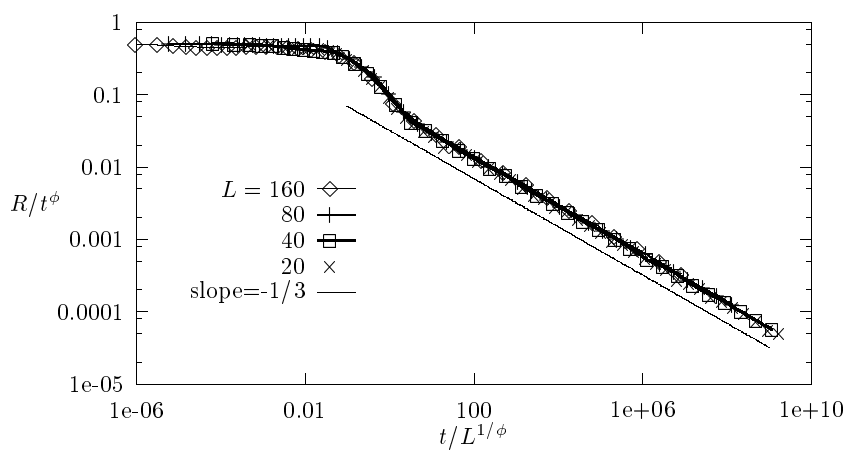

FIG. 3. Finite-size scaling plot of the characteristic length in the stress field, $R(t, L) . s=0.5, A=0.18$ with PBC.

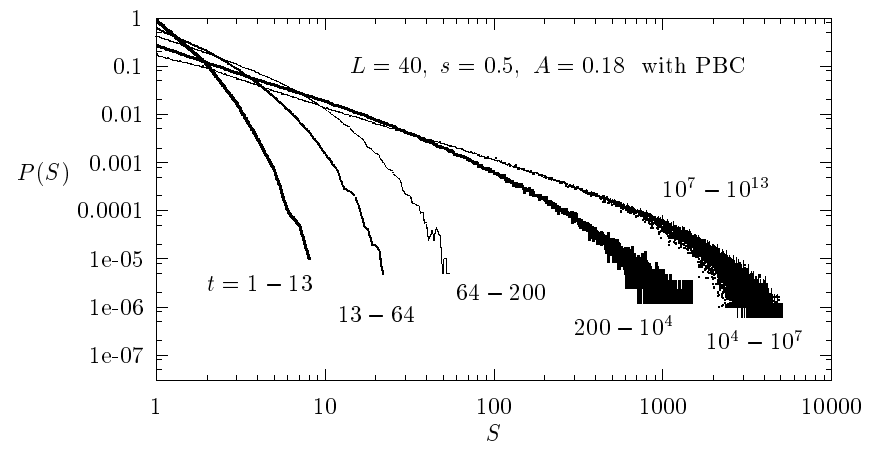

FIG. 4. $P(S)$ obtained from successive intervals in $t$ to show the approach to power law as $R(t) \rightarrow R_{0}$. 